\title{
Las publicaciones psiquiátricas nacionales y sus autores en 150 años de la especialidad: los primeros cincuenta años (1852-1902) Psychiatrics nationals publications and authors during 150 speciality years: The firsts fifty years (1852-1902)
}

\author{
Enrique Escobar M.
}

This work is a brief historical investigation with double objective: to recall the importants alienists who took care of the mental patients and too communicate its mains articles, emphasizing its communication by the importance epidemiologist and the clinica relevance of the commented subjects. This an effort work in the House of Orates, and after in the Manicomio Nacional developed next to sacrificed civil employees it was an wasp to get the paper living experiences in one site -as some them they proclaimed-with very bad and deplorable conditions.

Key words: History, authors, firsts chileans psychiatrics publications. Rev Chil Neuro-Psiquiat 2014; 52 (4): 274-280

\section{Introducción}

$\mathrm{E}$ la Biblia se describen enfermedades mentales sin hacer una diferencia clara entre sus causas religiosas, demoníacas o mágicas, siendo sustentados sus tratamientos en principios y prácticas varias: religiosas, interpretación de los sueños, musicoterapia, etc. ${ }^{1}$.

En la época grecorromana se describen las enfermedades mentales en libros como el Corpus Hippocraticum elaborada por Hipócrates y otros autores, postulando en la teoría de los humores que el desequilibrio de ellos (sangre, flema, bilis amarilla, bilis negras) son la causa de las enfermedades mentales y físicas. Dentro de las enfermedades mentales describen las grandes entidades nosológicas, entre ellas: el frenesí, la letargia, la manía y la melancolía causadas por alteraciones de los humores, teoría que con variaciones persistirá hasta el renacimiento y la ilustración en Occidente $^{2}$.

Fue Wilhelm Griesinger quien en 1845 afirmó que la causa de la enfermedad mental se debería a enfermedades cerebrales, si bien no descartó motivos psicológicos en algunas ${ }^{3}$. La especialidad médica que hacia 1802, Johann-Christian Heinroth (1773-1842) y Johann-Christian Reil (1759-1813) llamaron en alemán "psychiaterie", nuestra actual psiquiatría, nació a inicios del siglo XIX cuando médicos y filósofos franceses y de lengua alemana modificaron la visión de la locura, la manía en griego, vigente en la cultura occidental desde la antigüedad, intercambiando sus ideas a través de sus obras fundamentales ${ }^{4}$.

En 1800, en plena revolución francesa, Philippe

Recibido: 30/09/2014

Aceptado: 24/11/2014

El autor no presenta ningún tipo de conflicto de interés. 
Pinel (1745-1826) publicó la primera edición de su Traité médico-philosophique sur l'aliénation mentale ou la manie que cuatro años después, se publicó en Madrid en castellano y pronto pasaría a América. En ésta serán comunicadas durante el siglo XIX en revistas especializadas temas preferentes como el aislamiento de los locos en hospitales, la descripción clínica, las proposiciones nosológicas y las recomendaciones terapéuticas ${ }^{5}$. Sin embargo, antes de la aparición en revistas y textos especializados surgen las primeras publicaciones en diarios, tesis y folletos que dan cuenta del estado de desarrollo de esa ciencia, las condiciones clínicas y epidemiológicas de la época, la calidad de vida de los pacientes internados, etc. El primer trabajo conocido en Latinoamérica sobre psiquiatría apareció el viernes 29 de julio de 1791 en la Gazeta de Jamaica, y en Cuba fue reproducido el domingo 2 de octubre de 1791 en el Papel Periódico de La Habana con el título de "Idea curiosa" en el cual se sugería la inmersión bajo el agua como recurso terapéutico contra la locura ${ }^{6}$. En Chile también fue en diarios donde se informó en 1857 el caso de la endemoniada de Santiago, la Carmen Marín que por sufrir ataques atípicos en 1857 fue examinada por sacerdotes y médicos entregando cada uno su informe: posesión o enfermedad. De esa opinión fue el Dr. Manuel Carmona quien consideró que se trataba de una histeria ${ }^{7,8}$. Un mayor conocimiento del caso se encuentra en el texto de Roa ${ }^{10}$.

Esta comunicación es la primera de un estudio donde esperamos revisar las publicaciones psiquiátricas nacionales más importantes de 150 años de la especialidad. Este trabajo descriptivo de los primeros cincuenta años, informa sobre aspectos biográficos de los escasos alienistas chilenos, y comenta algunas de sus publicaciones. Esos primeros médicos -interesados también en la locura- trabajaron en su mayoría en La Casa de Orates, la única institución psiquiátrica nacional existente en la capital entre 1852 y 1902.

Nuestros alienistas educados en la corriente positivista trabajaron con pacientes internados en un espacio cerrado para tranquilidad de la comunidad, donde recibían protección y tratamiento dentro del contexto filantrópico imperante ${ }^{11}$. La información obtenida servirá además a futuras investigaciones de distintas disciplinas, como por ejemplo la historia y la psiquiatría. Utilizamos información obtenidas desde fuentes orales, escritas y computacionales.

\section{Desarrollo}

La fundación de la Casa de los Locos Nta. Sra. María de los Ángeles en 1852 a cargo del arquitecto Fermín Vivaceta señala el comienzo de la Psiquiatría Chilena. Ubicada primitivamente en el barrio Yungay se hizo inhabitable por el hacinamiento progresivo, debiendo el gobierno construir un mejor establecimiento -al norte del Río Mapocholigeramente afuera de la ciudad que contaba con cien mil habitantes. El nuevo edificio fue inaugurado definitivamente en 1858, en los mismos terrenos de la Avda. La Paz donde han seguido construyéndose sucesivas instalaciones para los enfermos mentales ${ }^{12-14}$. Después de dos años de abierta la Casa -administrada por miembros de la élite- se contrató al Dr. Lorenzo Sazié, francés, nacido en Assan, que estudió Medicina en los hospitales Necker, Saint Louis y Hotel Dieu con H. Dupuytren, F. Magendie, F. Broussais y J. Valpeau. En nuestro país ejerció como profesor de Obstetricia y por su capacidad creativa y habilidad organizativa, no exenta de un marcado rigor, inclinó al Gobierno a elegirlo como el primer decano de la Facultad de Medicina en 1843. Atendiendo que L. Sazié se había interesado en la patología mental, se le ofreció en 1854 un contrato parcial de médico de la Casa de Locos, donde fue ayudado por el primer practicante don Juan Ubeda y enseñó también durante un tiempo sobre las enfermedades mentales hasta poco tiempo antes de su fallecimiento en $1865^{15,16}$. Los trabajos conocidos de Sazié publicados en diarios de la época más bien de salud pública no los comentamos acá17,18.

A fines de los cincuenta en vista del crecimiento de la población se contrató para ayudar al Dr. Sazié al Dr. Ramón Elguero del Campo, argentino, nacido en 1819, hijo de Sabina Cuadra y Antonio Elguero. Este último, militar, integrante del Ejér- 
cito Libertador volvió a Argentina, y fue fusilado en 1842, por orden del Dictador Rozas. Elguero ingresó en 1837 al Instituto Nacional, donde enseñó latín y luego empezó sus estudios de Medicina, carrera que interrumpió, pues su nombramiento de profesor de patología interna, dada la escasez de académicos, generó seria incomodidad entre los estudiantes. Decidió viajar a Valdivia, aceptando un cargo como cirujano del ejército y Rector del Liceo de la ciudad. En Valdivia incursionó en la política, adhiriendo al Partido Nacional o Monttvarista que impulsaba el progreso económico y la educación, siendo elegido Diputado por dos períodos en representación de Valdivia y posteriormente de Osorno en 1852, 1854 y 1861 respectivamente. Ya en Santiago, en 1853, se licenció con una tesis sobre las enfermedades del corazón e inició su trabajo en el Hospital San Borja y luego en el San Juan de Dios, el que combinó con su dedicación a la Casa de Orates donde enseñó transitoriamente psiquiatría -constituyéndose en el primer profesor de la especialidad- retirándose por enfermedad cerebro vascular en 1864, y falleciendo en $1877^{18}$. Augusto Orrego Luco, alumno de Elguero, opinaba que Elguero fue un hombre de gran valor moral, orgulloso y reservado, que a su juicio sería uno de los últimos médicos que leía a los clásicos en latín y que su mayor interés fue la psiquiatría. Termina afirmando que fue tratado injustamente, ya que nunca se le reconocieron sus méritos, siendo "víctima de la mediocridad, rodeado de silencio y vacío por una sorda y secreta hostilidad"19. Entre las comunicaciones del Dr. Elguero destacamos un estudio estadístico sobre la Casa de Orates, conocido como "Informe del Médico de la Casa de Locos" donde comunica una investigación epidemiológica descriptiva del movimiento censal de los internos entre 1860 y 1862, destacando un aumento constante de la internación consecuencia principalmente del alcohol, la herencia y la degeneración. Sin embargo, mayor interés suscita la discusión sobre la descripción de la nosología y los tratamientos imperantes en aquella época. Elguero conocía seguramente los planteamientos de V. Chiarugí, $P$. Pinel y J.E. Esquirol pues al iniciar su comunicación refiriéndose a la clasificación alude que "por ser una cuestión de lo más controvertida algunos alienistas pretenden que sólo debe atenderse a los síntomas de la locura, mientras que otros estiman como de más valor la causa próxima o inmediata de la locura". Más adelante continúa: "necesitando optar entre ellas para dar principio a mi trabajo, acepto la clasificación que tiene por base los caracteres sintomáticos, a pesar de las muchas dificultades que ofrece en su aplicación". Su propuesta, simple en comparación con las actuales nosologías distingue: Manías (Agudas y Crónicas), Monomanías (Lipemanías o Melancolía, Hipocondría Religiosa, Erotomanía), Demencia Simple y Epiléptica, Imbecilidad e Idiocia. Posteriormente al comentar sobre los tratamientos, vuelve a traslucirse su actitud ecléctica "Yo, por mi parte, no me adhiero exclusivamente a ideas preconcebidas, ni soy partidario de ningún sistema que se base en la exclusión de otros. Yo acepto todas las medicaciones racionales: pues según sean las condiciones particulares que ofrezcan los individuos, pueden dar, solas o reunidas, resultados eminentes, los cuales jamás se obtendrían si se emplearan por separado los métodos expresados". De este modo cita la sangría, los purgantes, los baños y varios fármacos (opio, belladona, hachisch, etc.), agregando enfáticamente la importancia de la terapia moral en el asilo terapéutico que incluye la persuasión, la disciplina, la separación de la familia, la contención, los baños, el régimen alimenticio, etc. ${ }^{20}$.

En reemplazo del Dr. Elguero fue contratado en 1875, como médico residente el Dr. Guillermo Benham de nacionalidad inglesa, quien al constatar las condiciones de pobreza, hacinamiento y abandono de recursos humanos y materiales de la Casa procedió a informar al gobierno lo que generó el repudio de la administración del hospital y su rechazo a reanudar su contrato de cuatro años, alegando incumplimiento laboral. El Dr. Benham, ardiente defensor de la terapia moral, falleció poco después en $1879^{21}$.

A continuación se contrató al Dr. Carlos Sazié Heredia, hijo del Dr. L. Sazié, nacido en 1852, quien luego de sus estudios en el Instituto Nacional y en la Universidad de Chile, viajó a especializarse a Francia gracias a una beca del Estado. Durante su 
formación desde 1874 a 1879 aprendió con V. Magnan, F. Voisin y J.M. Charcot y su tesis de doctorado "Problemas Intelectuales en la Afasia" fue patrocinada por el Dr. Félix Vulpian, neurólogo y en ese momento Decano de la Facultad de Medicina de Paris. De vuelta en Chile se ofreció a trabajar gratis en el hospital manifestando su deseo de compensar al Estado por haberle financiado su especialización $^{22}$. Además contratado por la Universidad de Chile como segundo profesor de enfermedades nerviosas y mentales, Sazié había iniciado un curso para estudiantes de medicina en la Casa de Orates donde trabajó y enseñó hasta 1884, momento en que fue rescindido su contrato por desacuerdos con la administración como luego veremos. Sazié continuó ejerciendo la docencia como profesor titular de la Cátedra de Enfermedades Nerviosas y Mentales en el hospital San Juan de Dios y luego en el nuevo Hospital San Vicente de Paul. En cuanto a sus publicaciones, tenemos "La Anestesia Histérica y los Agentes Estesiógenos" donde describe la sintomatología y discute los tratamientos para influir en la sensibilidad anormal, parestesia y otros síntomas histéricos con el cloral, los bromuros, el paraldehido, las infusiones aromáticas, la electroterapia y la hipnosis ${ }^{23}$. También refutaba al Dr. Ramón Araya Echeverría, quien en el trabajo Electroanestesia había comunicado la invención de una máquina para tratar a las pacientes histéricas ${ }^{24}$. Según Araya bajo la influencia de una acción específica de la corriente provocaba insensibilidad terapéutica hecho que Sazié explicó que era por efecto de una sugestión hipnótica. En otro artículo "Influencia del trabajo i de las distracciones en el tratamiento de la enajenación mental" propuso cambios en el tratamiento de los pacientes mediante la rehabilitación laboral, con labores agrícolas y con talleres que fueron desechados por la administración y motivo como arriba señalamos para ser despedido de la Casa de Orates ${ }^{25}$. Sazié, simpatizante del Presidente Balmaceda, fue despedido de la Universidad de Chile después del triunfo de la Revolución de 1891 -turbas fanáticas incendiaron su Sanatorio Neuropsiquiátrico privado- permaneciendo exiliado en Francia y vuelto a Chile se radicó en Quilpué donde falleció en 1921.
Continuando con la revisión emprendida surge la figura destacada del Dr. Augusto Orrego Luco quien nació en 1848 en Valparaíso, estudió en el Instituto Nacional. Por decisión de su padre entró a estudiar Leyes, pero a escondidas también inició la carrera de Medicina graduándose con la memoria "Causas indirectas de la alucinación mental" en 1884. Fue casado con Martinica Barros Borgoño mujer intelectualmente dotada, que era la primera en escuchar los trabajos de su esposo y fundó una tertulia cultural donde asistían personajes importantes de la sociedad chilena ${ }^{26}$. Una vez egresado, ejerció la docencia en Anatomía hasta partir a Francia a especializarse con Jean Martin Charcot en París y Wilhelm Erb en Alemania. Vuelto a Chile enseñó en la Universidad enfermedades nerviosas y mentales bajo el alero de la nueva Cátedra desde 1892 a 1905 alcanzando el cargo de Director de la Escuela de Medicina. Junto con ejercer la medicina y la docencia destacó como escritor, historiador y político. Trabajó un breve tiempo en el Hospital Psiquiátrico y dedicó su vida a la educación y a la vida pública ya sea en el aula, en los medios periodísticos, en el Parlamento, etc. ${ }^{27}$. Fue un activo colaborador de la Revista Médica y del diario La Patria donde comunicó varios trabajos entre ellos algunos referidos a la Cuestión Social asunto que dejaba traducir su ideología liberal y su preocupación por la alta morbilidad y mortalidad infantil. Su dedicación a su profesión y su capacidad clínica no pasaron desapercibida a los pobres que atendía en el hospital San Vicente de Paul ganándose el apodo "el mago de la Cañadilla" por su habilidad diagnóstica. De sus variados escritos donde demostró su elocuencia y destreza destacamos dentro del campo de la psiquiatría sus estudios sobre el método de exploración anátomo-clínico, el diagnóstico diferencial de la hemiplejía histérica y orgánica, las alucinaciones mentales, las circunvoluciones del cerebro, las neurosis mímicas, etc. En el primero enfatiza el cuidadoso examen acentuando la importancia de la anatomía y naturalmente los síntomas mentales. Su texto más destacado publicado en 1904 fue la Etiología y Terapéutica de la Tabes Dorsal donde adhiere a la opinión de Fournier y Erb respecto a la causa luética de la Tabes. Se trata 
de un libro de 146 páginas donde estudia a una suficiente población de Tabéticos Dorsales aplicando el método crítico clínico de su invención ${ }^{28}$. Agreguemos que en letras, nos dejó su prestigiado libro "Recuerdo de la Escuela" donde describe con nostalgia y cariño la vida estudiantil y al recordado profesor Ignacio Domeyko y el otro texto sobre la historia antigua de la patria ${ }^{29,30}$. El profesor Orrego Luco falleció en 1933.

En la última década del siglo XIX apareció el Dr. Manuel Beca Pérez, hijo de don Manuel y Doña María del Pilar nacido en 1863 en Ancud, donde luego de terminar sus estudios de humanidades, viajó a Santiago para estudiar en la Universidad de Chile, donde se graduó a los 23 años. Casado con Doña Rosa Soto, tuvo dos hijos ${ }^{31}$. Beca trabajó en la Casa de Orates y fue Director de la Sociedad Médica de Santiago donde comunicó la mayoría de sus trabajos. Participó en diversos congresos médicos, asistiendo incluso en el extranjero al Congreso de Alienistas franceses de 1895 en Burdeos donde dentro de los asistentes cita a reputados colegas como J. Moreau, J. Seglás, J. Babinski, A. Delmás, S. Korsakoff, etc. y en el V Congreso Penitenciario Internacional de Paris del mismo año, donde se recomendó la fundación de establecimientos especiales para los criminales enajenados ${ }^{32}$. Falleció en 1919 habiendo publicado varios artículos dedicados a la salud mental, el alcoholismo, la administración del hospital de locos, etc. Su tesis para licenciarse "Algo sobre las enfermedades mentales en Chile" publicado en 1885, seguido por su trabajo "Contribución al estudio de las enfermedades mentales en Chile" de 1891 dieron origen a la costumbre de publicar anualmente las Memorias de la Casa de Orates de Santiago que guardan en la Biblioteca del Instituto Psiquiátrico ${ }^{33}$. Por último, conviene agre- gar que durante las primeras décadas la institución hospitalaria adolecía de recursos físicos y humanos agrabados por el hacinamiento, denunciados por algunos médicos que por lo mismo sufrieron el despido. Sin embargo, en las últimas décadas luego de un informe oficial de los Drs. José Joaquín Aguirre y Octavio Moore se decidió a fines del siglo XIX, la construcción de una institución moderna -dotada inclusive de una incipiente bibliotecacircundadas de parques, el Manicomio Nacional, consistente en un amplio edificio de dos pisos con entrada por la calle Olivos ${ }^{34,35}$.

\section{Conclusión}

Las enfermedades mentales están descritas desde la antigüedad, afirmando que sus causas transitan desde creencias religiosas, mágicas y diabólicas. La visión científica aparece a fines del siglo XIX y comienzos del siglo XX. La publicación de patología mental en algunos países, aparece primero en diarios antes que en revista y libros. Así fue en Chile, donde en 1857 se publicó un comentado caso de Histeria en varios diarios de la época. Los escasos alienistas reconocidos procedentes de la incipiente clase media y oligárquica muestran conocimientos adecuados, fundados en revistas especializadas extranjeras e incluso algunos con estudios en Europa $\mathrm{y}$, por lo tanto, con una competencia de acuerdo con su época. La revisión de los escritos psiquiátricos chilenos, durante el período investigado, revela que fueron publicados al comienzo en diarios y pequeños impresos hasta que apareció la Revista Médica de Chile en 1872. Esas publicaciones versan sobretodo en la descripción de casos clínicos, propuestas de nosologías e investigación estadística de los pacientes internados. 


\section{Resumen}

Se trata de una investigación histórica con un doble objetivo: recordar a los alienistas chilenos que atendieron a los enfermos mentales y comunicar algunos de sus artículos relevantes clínica y epidemiológicamente, publicados en los primeros cincuenta años de la psiquiatría chilena. Durante esa esforzada época-desarrollada en la principal institución nacional-progresivamente fue consolidándose la especialidad hasta ser conocida en el extranjero. El esforzado trabajo desarrollado en la Casa de Orates y luego en el Manicomio Nacional, junto a los sacrificados funcionarios, fue probablemente un aguijón para llevar al papel las experiencias vividas en un lugar-como algunos lo denunciaron-de pésimas y deplorables condiciones.

Palabras clave: Historia, autores, primeras publicaciones psiquiátricas chilenas.

\section{Referencias bibliográficas}

1. Halpern-Zaoni B,Winner H. La psiquiatría en la civilización hebrea antigua en Postel J. Quétel J (coordinadores) Nueva Historia de la Psiquiatría Fondo de Cultura Económica, México, Segunda Edición 2000 p 39-45.

2. Gourevitch D. La psiquiatría en la antigüedad Grecorromana en opus. cit. p 23-36.

3. Griesinger W. Biografía en opus. cit. p 648-9.

4. Villaseñor S, Rojas C. Garrabé J Editores Antología de textos clásicos de la psiquiatría latinoamericana Gladet citado en http://www.gladet.org.mx// publicaciones/textosclásicos de psiquiatralatinoamericano.pdf consultado el 1 de noviembre de 2014.

5. Mariátegui J. Prensa psiquiátrica latinoamericana Revista de Neuro-Psiquiatría del Perú-Tomo LXIII septiembre-diciembre, No 3-4 2000 citado en http://sisbib.unmsm.edu.pe/bvrevistas/neuro_psiquiatría/v63_3-4prensa psiquiatra.htm consultado el 1 de noviembre de 2014.

6. López Espinoza JA. Primeras publicaciones cubanas sobre psiquiatría ACIMED 2001; 9 (3): 1-4.

7. La Endemoniada de Santiago. El Mercurio de Valparaíso del 3 de agosto de 1857.

8. El Ferrocarril de Santiago del 4 de agosto de 1857.

9. Roa A. Demonio y Psiquiatría: aparición de la conciencia científica. Editorial Andrés Bello, Santiago 1974.

10. Aroca A. Historiografía de la locura. El péndulo de la historia Universidad Libre de Bruselas, Revista Observaciones Filosóficas 2010; 10: 26-29.

11. Actas de la Junta Directiva 18541891 Casa de Orates de Santiago. Imp. Valparaíso 1901. Santiago de Chile.

12. Escobar E. Historia del Hospital Psiquiátrico (1852-1952). Rev de Psiquiatría 1990; 7 (1):361-8.

13. Medina E. Panorama Institucional de la Piquiatría Chilena. Rev Psiquiatría VII 1990; 7 (1): 343-60.

14. Escobar E. Lorenzo Sazié Primer Médico de la Casa de Orates. Rev Chil Neuro-Psiquiat 1989; 53 (37) 3: 186-8.

15. Escobar E. Medina E. Morlans I. Historia del Instituto Psiquiátrico Dr. José Horwitz en La Psiquiatría en Chile Apuntes para una Historia. Armijo A. Editora General 2010 Santiago, Chile.

16. Sazie L. Ojeada sobre la causa de algunas enfermedades graves y sobre la estadística médica de Chile en Diario El Progreso, No 343, Santiago, 25 de diciembre de 1843.

17. Sazié L. Sobre las muertes aparentes en Revista de Santiago, 1849; t. III, 169-78.

18. Escobar E. José Ramón Elguero del Campo (18191877). Rev Med Chile 2000; 54 (2): 131-2.

19. Orrego Luco A. Recuerdos de la Escuela Editorial del Pacífico 1953. Santiago, Chile.

20. Elguero José R. Informe del Médico de la Casa de Locos noviembre de 1862 ver Costa Casareto C. Ideario Psiquiátrico del Médico de la Casa de Locos de Santiago. Rev Med Chile 1980; 108: 559-6.

21. Benham W. Casa de Orates: Informe pasado al Mi- 
nisterio del Interior Santiago de Chile imprenta de la República (citado por Casta Cassaretto en op.cit 1980).

22. Escobar E. Carlos Sazié Heredia, segundo profesor de neurología y enfermedades mentales en Chile. Rev Chil Neuro-Psiquiat 2001; 39 (2): 165-6.

23. Sazié C. La Anestesia Histérica y los Agentes Estesiógenos. Rev Med Chile 1883; 9: 49-53.

24. Araya Echeverría R. La Electroanestesia. Rev Med Chile 1882; 2: 44-50.

25. Sazié C. Influencia del trabajo i de las distracciones en el tratamiento de la enajenación mental. Rev de Chile, Imprenta Gutenberg Tomo 1, 1881; 1, 17888.

26. Escobar E. Augusto Orrego Luco Fundador de la Cátedra de Neurología y Psiquiatría (1848-1933) Rev Chil Neuro-Psiquiat 2002; 56, 40, 1: 88-9.

27. Ruíz Torres A. La contribución de Augusto Orrego Luco a la psiquiatría chilena. Rev Med Chile 1996; 124: 124-6.
28. Orrego Luco A. Etiología y Terapéutica de la Tabes Dorsal Imprenta, Litografía y Encuadernación. Barcelona Santiago Chile 1904.

29. Orrego Luco A. Recuerdos de la Escuela Editorial del Pacífico Santiago Chile 1953.

30. Orrego Luco A. La Patria Vieja Prensas de la Universidad de Chile 1933.

31. Escobar EM. Beca Pérez (1863-1919) Rev Chil Neuro-Psiquiat 2003; 57, 41, 1: 34-7.

32. Beca M. Conferencia en Congreso de Alienistas y Neurologistas de Francia. Rev Med Chile 1895; 23 (8/9): 385-9.

33. Beca M. Algo sobre las Enfermedades Mentales en Chile Recopilación y Estudios de la Estadística de la Casa de Orates Imprenta Nacional 1885.

34. Aguirre JJ, Moure O. Informe de la Casa de Orates Junta de Beneficencias 1893.

35. Diario El Constitucional "Inauguración de la nueva construcción de la Casa de Orates" 16 de noviembre de 1896.
Correspondencia:

Enrique Escobar Miguel

Arturo Ureta 1511, Vitacura.

Teléfono: 222289265

E-mail: dreescobarm@gmail.com 